\title{
CalédoMax : la solution de la Nouvelle-Calédonie pour la mesure des hauteurs maximales de crues
}

\author{
Christophe LAROCHE ${ }^{1}$, Pearl WINCHESTER ${ }^{1}$, Nordan BERNAST ${ }^{1}$, Geoffroy WOTLING ${ }^{1}$, Nicolas ROMIEUX', \\ Christian DELEBRET ${ }^{2}$, Gilles RICAUD ${ }^{2}$, Bernard REY ${ }^{2}$. \\ 1. Service de l'Eau/Direction des Affaires Vétérinaires, Alimentaires et Rurales (DAVAR) - 209 rue Auguste Bénébig, Haut Magenta - \\ BP 256-98845 Nouméa Cédex, Nouvelle-Calédonie,prénom.nom@gouv.nc \\ 2. Retraité du Service de l'Eau de la DAVAR
}

\begin{abstract}
RÉSUMÉ. - Pour disposer en de nombreux endroits des cotes maximales de crues et ce à moindre coût, l'implantation d'échelles à maxima s'avère très utile. Après avoir passé en revue les principaux types d'échelles à maxima rencontrés dans la littérature, nous détaillons le système développé en Nouvelle-Calédonie, appelé CalédoMax. Ce système s'avère être largement original principalement dans sa manière de mesurer et mémoriser la hauteur maximale atteinte durant la crue. Il est constitué de deux cordes rugueuses tendues sur lesquelles coulisse un flotteur qui pousse une bague montée sur l'une des cordes. Lors de la montée des eaux, le déplacement du flotteur vers le haut pousse la bague vers le haut. Celle-ci restant en position maximale lors de la décrue puisque prise en tenaille entre les deux cordes, elle permet d'approcher le niveau maximum atteint par la crue. Ensuite, les principales utilisations des échelles à maxima passées en revue, nous mettons en avant celles que nous privilégions en Nouvelle-Calédonie et qui concernent l'hydrométrie. Puis nous établissons un retour d'expérience de l'utilisation de notre réseau de CalédoMax qui, depuis 1988 a pu compter jusqu'à plus de 900 appareils et qui a permis de réaliser plus de 16400 mesures. Enfin, nous présentons notre méthode de stockage et de gestion des informations ainsi collectées, avant de conclure sur les possibilités de répliquer le CalédoMax dans d'autres contextes ainsi que les améliorations qui pourraient être envisagées.
\end{abstract}

Mots-clés : échelles de crue, hydrométrie, crue, inondation, retour d'expérience

\section{CaledoMax: New-Caledonia's solution for maximum flood level measurements}

\begin{abstract}
Crest gauges are often used to record maximum flood level at minimal cost. Around the world, different cheap and easy to make instruments have been designed. In our article, we first establish a rapid benchmark of the different crest gauges used around the world. Next, we focus on the New-Caledonia's instrument named CalédoMax. This innovative measurement system is composed of two strings, a floating system and a ring. With the water level rising, the floating system rises, pushing up the plastic ring, itself sliding between two strings. When the water level decreases, the floating system slides down, but not the ring, which stays stuck between the two strings. The ring is then the tracker of the highest water level reached by the flood that we can easily measure after the flood.

With more than 900 CalédoMax installed since 1988, to this date 16400 measurements have been fulfilled. A database has been created to cluster these measurements and is updated after every new flooding episode. This database helps the identification and analysis of the main problems met on the instruments and the improvements that can be made in the use of the Caledomax. The system is rather sturdy, with some of the CaledoMax being installed and working for almost 30 years without trouble. But it is necessary to keep regular maintenance and field visits, as for any measurement instrument. The database also facilitates the use of the data for hydrological purposes, like hydraulic modelling for example.

When installed around a hydrometric station, the CaledoMax adds extra water level data in flooding conditions to the regular continuous water level recording. Finally, we discuss the possible improvements for the CaledoMax system and how it could be used it in other environments.
\end{abstract}

Key-words: flood crest gauges, hydrometry, flood, feedback, lessons learnt

\section{INTRODUCTION}

Pour apprécier la gravité d'une grande inondation, au-delà des mesures disponibles aux stations hydrométriques, il est d'usage de rechercher les niveaux maxima atteints en différents endroits du lit majeur. Si l'on dispose de ces informations en différents endroits et pour différentes crues, il est alors possible d'apprécier la gravité de chacune de ces crues. En outre, l'importance de disposer de ces niveaux maxima atteints est évidente pour le calage des modèles hydrodynamiques qui se basent en grande partie sur la comparaison de ces informations avec les cotes calculées. Ce contexte a motivé récemment la mise en place d'une base de données collaborative ${ }^{1}$ recensant, à l'échelle nationale, les repères de crues existants [Piotte et al., 2016], ce qui permettra à chacun, de disposer de l'ensemble des informations disponibles sur les crues historiques.

1. https://www.reperesdecrues.developpement-durable.gouv.fr/, consulté le 9/05/2017 
Pour apprécier la gravité des crues futures, cette stratégie de collecte des cotes maximales de crues peut suffire dans bien des territoires. Toutefois, elle ne garantit pas de disposer d'une estimation raisonnable de ces cotes maximales, notamment dans des territoires peu urbanisés voire ruraux, où les repères servant de référence sont difficiles à identifier et à pérenniser. La mise en place de réseaux d'échelles à maxima de crues participe de cette volonté de garantir en des endroits judicieusement choisis, même en pleine nature, la collecte de niveaux maxima atteints lors des futures crues.

\section{LES TYPES D'ÉCHELLE À MAXIMA RENCONTRÉS DANS LA LITTÉRATURE}

Il existe différents types d'échelle à maxima. L'appareil le plus couramment commercialisé en France métropolitaine est sans aucun doute l'échelle limnimétrique équipée d'un ruban à décoloration présenté en figure $1 \mathrm{a}$, le tout placé dans un tube en plexiglas transparent et résistant, de diamètre d'environ $50 \mathrm{~mm}$. Son principe de fonctionnement est simple : l'eau en montant dans le tube contenant l'échelle limnimétrique efface la couleur du ruban jusqu'au niveau atteint ${ }^{2}$. Un filtre, situé à l'intérieur du pied du cylindre, permet de retenir les plus grosses impuretés de l'eau et joue le rôle d'amortisseur des trop importantes oscillations pouvant exister à la surface libre des écoulements très rapides. D'autres dispositifs ont été imaginés à travers le monde dont le dénominateur commun semble être le tube d'un diamètre de l'ordre de $50 \mathrm{~mm}$ dans lequel est placé le système de mesure. Par exemple le service hydrologique du Maroc a placé dans un tube galvanisé une échelle (ou un simple élément de zinc peint en blanc) recouverte d'une peinture

2. http://www.ott.com/fr-fr/produits/le-niveau-deau-72/echelle-a-maximum-ott-191/, consulté le 9/05/2017 lavable de couleur [Nouvelot, 1993], qui sera ainsi lessivée par la crue. Deux autres systèmes de mesure aussi recensés par Nouvelot [1993] sont constitués l'un de copeaux de bois ou de débris de polystyrène placés en pied du cylindre et qui sont entraînés par l'eau jusqu'à se déposer dans des alvéoles réparties tout au long du tube, l'autre d'un système de godets, dans lesquels sont déposés de petits flotteurs en bois qui seront déplacés par l'eau. Une variante de ce système consiste à disposer dans les godets de petits flotteurs en plastique qui seront déplacés durant la crue. Ce système a été observé ${ }^{3}$ sur l'étang de Pierre Blanche ${ }^{4}$, dans le Sud de la France. La figure $1 \mathrm{~b}$ présente cet appareil.

L'utilisation de copeaux de liège ou de polystyrène a aussi été retenue dans le système décrit dans USGS [2010] ainsi que partiellement dans OMM [2008]. Placés initialement dans un réservoir en pied du tube, les copeaux flottent et adhèrent à une tige de bois graduée située au centre du tube, lors de la redescente du niveau d'eau. Le pied du tube, ainsi que le bouchon fermant son sommet, sont percés afin que l'eau et l'air puissent circuler facilement à l'intérieur du tube. Ce système, présenté en figure 1c semble être largement utilisé aux États-Unis et commercialisé avec des tubes de plusieurs diamètres en acier galvanisé ou en $\mathrm{PVC}^{5}$. En France, Électricité de France disposait jusqu'à très récemment de quelques appareils similaires utilisant les copeaux de liège et une règle graduée à l'intérieur d'un tube fendu sur toute sa longueur pour que l'eau puisse y pénétrer ${ }^{6}$. La face fendue du tube devait probablement être placée à l'opposé des courants principaux.

\footnotetext{
3. Marchand $\mathrm{P}-$ Communication personnelle

4. Le gestionnaire de ce système est le Syndicat du Bassin du Lez (syble.fr)

5. http://rickly.com/new-category-46/?sort=featured\&page=1, consulté le 9/05/2017

6. EDF-DTG, Département Surveillance, Service Environnement
} Aquatique - Communication personnelle

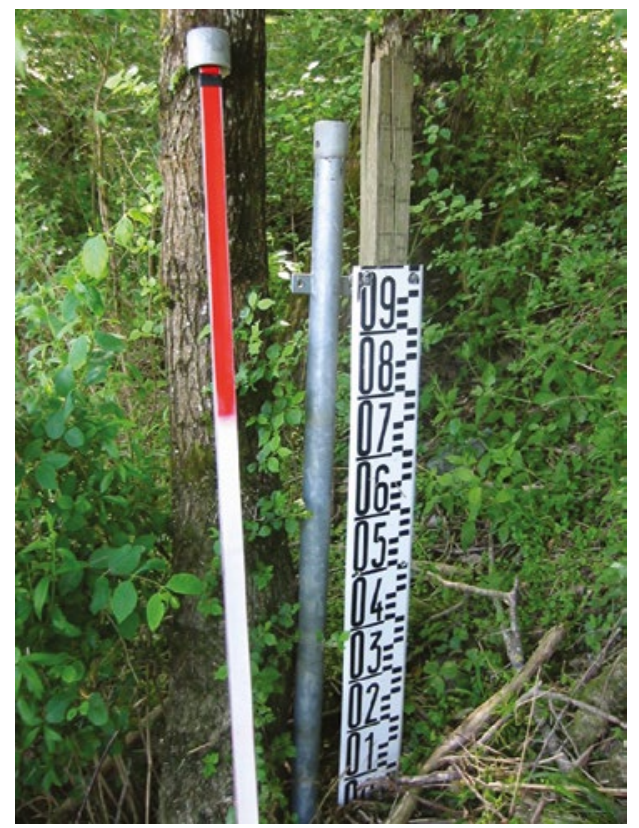

a) Ruban à décoloration monté sur support métallique (source G. Dramais IRSTEA).

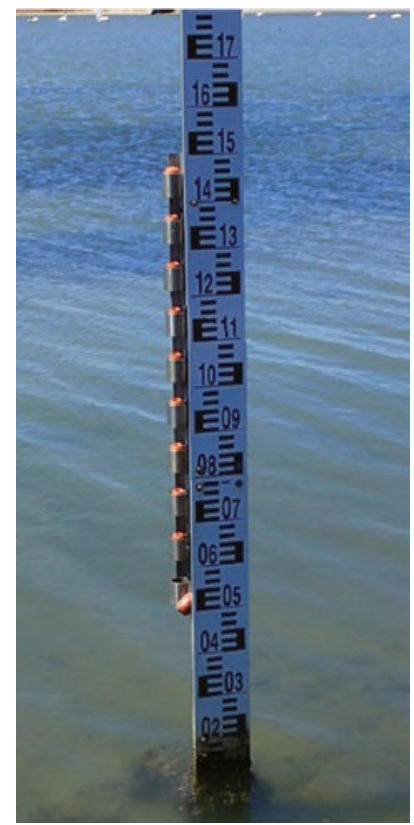

b) Système à godets et flotteurs (source P. Marchand, IRD).

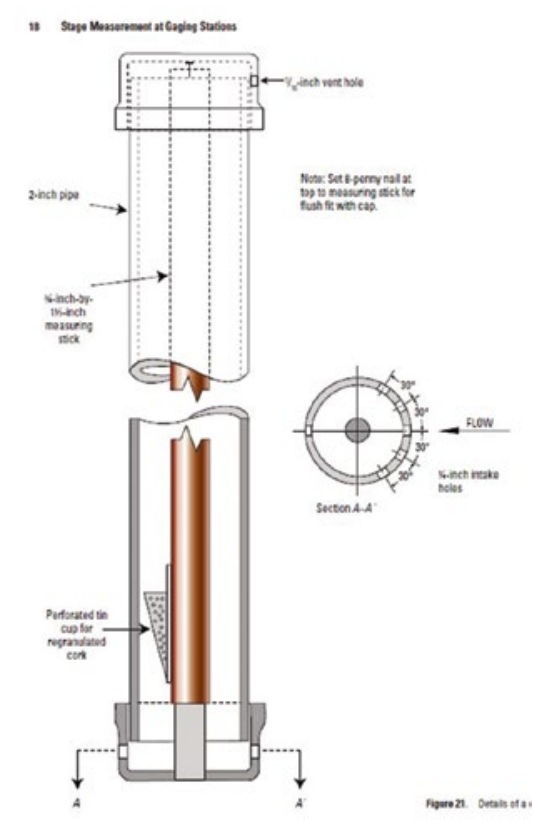

c) schéma de principe du système à sciure de bois [USGS, 2010].

Figure 1 : Différents systèmes d'échelles à maxima de crue. 
Ensuite, dans Zaher [2017] citant Roose [2000], il est décrit l'utilisation d'une échelle constituée d'un fer à cornière de $50 \mathrm{~mm}$ de côté, sur laquelle sont montés des godets tous les 5 centimètres dans lesquels l'eau sera conservée après le passage de la crue. Ce dispositif simple et original nécessite toutefois d'être en mesure de relever le niveau d'eau mesuré rapidement après la crue avant que l'évaporation ne l'efface.

En Nouvelle-Calédonie, le dispositif imaginé il y a une trentaine d'années, s'appelle le CalédoMax. Bien que celui-ci soit aussi disposé dans un tube de diamètre $50 \mathrm{~mm}$ environ, l'originalité de sa conception réside clairement dans son système de mesure et d'enregistrement de la hauteur d'eau, simple à réaliser, fiable, particulièrement robuste et qui a fait preuve d'une grande longévité.

\section{PRÉSENTATION DU PRINCIPE DU CALÉDOMAX}

Dans les années 1980, 3 agents de la DAVAR ont imaginé une échelle à maxima grâce à un appareillage simple et astucieux. Ce système a bien entendu nécessité plusieurs tests avant d'aboutir au système tel que présenté ci-après, notamment dans le choix des matériaux le constituant.

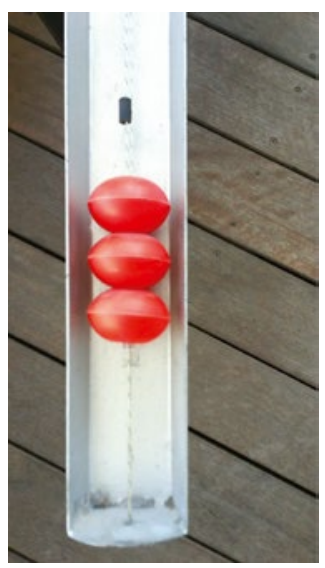

A l'instar des principaux systèmes présentés ci-avant, il se décompose en deux parties, la première est constituée du mécanisme d'enregistrement de la hauteur maximale de l'eau à l'aide d'un flotteur de filet de pêche (en rouge sur la figure 2) monté sur un tube plastique (i.e. corps de stylo à bille) et se déplaçant le long d'un ensemble de deux cordes. Celles-ci sont tendues dans un cadre métallique appelé réglette à l'aide d'un simple domino d'électricien. La réglette est obtenue par plusieurs pliages d'une feuille d'acier galFigure 2: Système d'enregistrement de la hauteur maximale de la crue équipant le CalédoMax. pour la pêche en mer profonde. Sur l'une des deux cordes est montée une bague en plastique (en noir sur la figure 2) qui est poussée vers le haut par le flotteur. Cette bague est ainsi comme prise en tenaille par les deux cordes tendues. Il faut souligner ici que les cordes étant tressées, cela les rend suffisamment rugueuses pour conserver la bague, par ailleurs cannelée, dans sa position maximale, lorsque le flotteur redescend en décrue. Ainsi le niveau d'eau maximum atteint lors de la crue est donné par la position de la bague.

La figure 3 présente la séquence conduisant à l'enregistrement par le CalédoMax d'une hauteur d'eau.

La seconde partie du système représente le logement du système d'enregistrement de la hauteur d'eau maximale, il est classiquement composé d'un tube en acier galvanisé. La base de celui-ci est percée de multiples trous qui, associés à un filtre de graviers disposé sur le pourtour du tube, permettent son remplissage et sa vidange sans introduire de corps flottant pouvant fausser les déplacements du flotteur et de la bague. Ce système permet, dans les conditions supposées de régime permanent, d'approcher la charge hydraulique de l'écoulement puisque alors plus aucun écoulement ne s'opère dans le tube. Ce système d'alimentation en eau du tube est sans aucun doute moins sensible aux variations rapides de niveau d'eau extérieur (vagues) que celui utilisé par l'USGS, basé sur un écoulement à travers une ou plusieurs séries d'orifices situées le long du tube. En outre, pour ces derniers, des tests en laboratoire par Firday [1965], ont montré qu'il pouvait exister une perte de charge entre l'intérieur et l'extérieur du tube de quelques centimètres, celle-ci dépendant des vitesses d'écoulement, du nombre de séries de trous et de leur position par rapport à l'écoulement. Malgré ce filtre de graviers, il a été nécessaire d'ajuster la conception du flotteur du CalédoMax afin que celui-ci ne reste pas collé en position basse lors d'une crue du fait des limons séchés qui entrent malgré tout dans le tube. Pour cela, le nombre de flotteurs est rapidement passé à trois, comme présenté en figure 2 .

Le tube galvanisé est fiché dans une embase en acier elle-même ancrée dans le sol à l'aide d'un massif en béton (figure 4). Le tube est fixé à l'embase grâce à une cheville acier et un boulon, ce qui permet le remplacement aisé du CalédoMax en cas de dysfonctionnement. Sa tête est quant à elle fermée par un capot verrouillé à l'aide d'une clé Allen. Sur ce capot est fixée une plaque signalétique présentant le numéro du CalédoMax (figure 5).

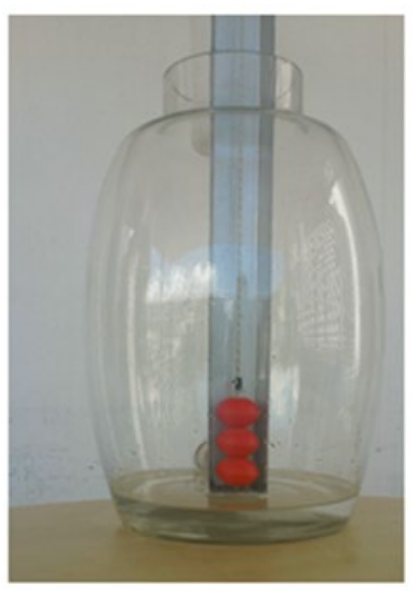

a)

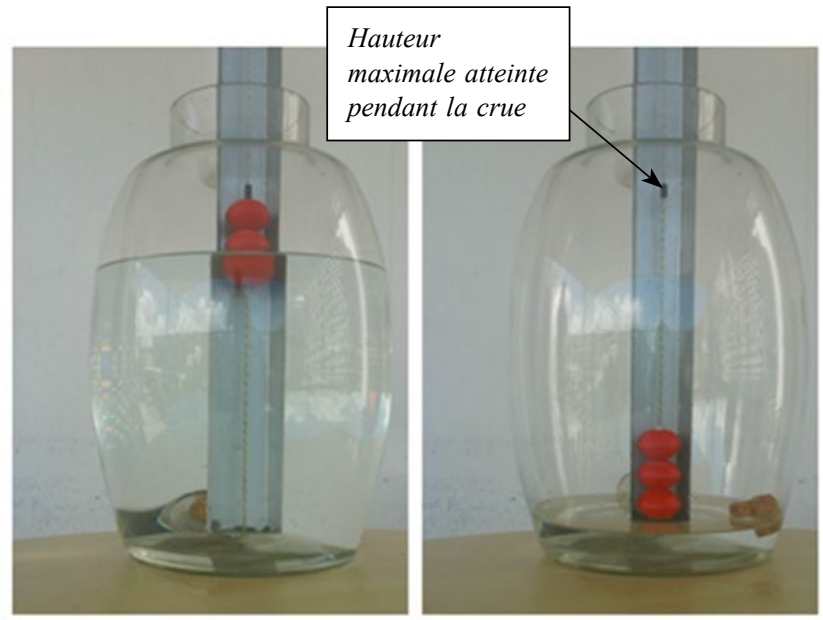

c)

Figure 3 : Déplacements du flotteur et de la bague : position initiale (a), lors de la montée (b) et après la crue (c). 


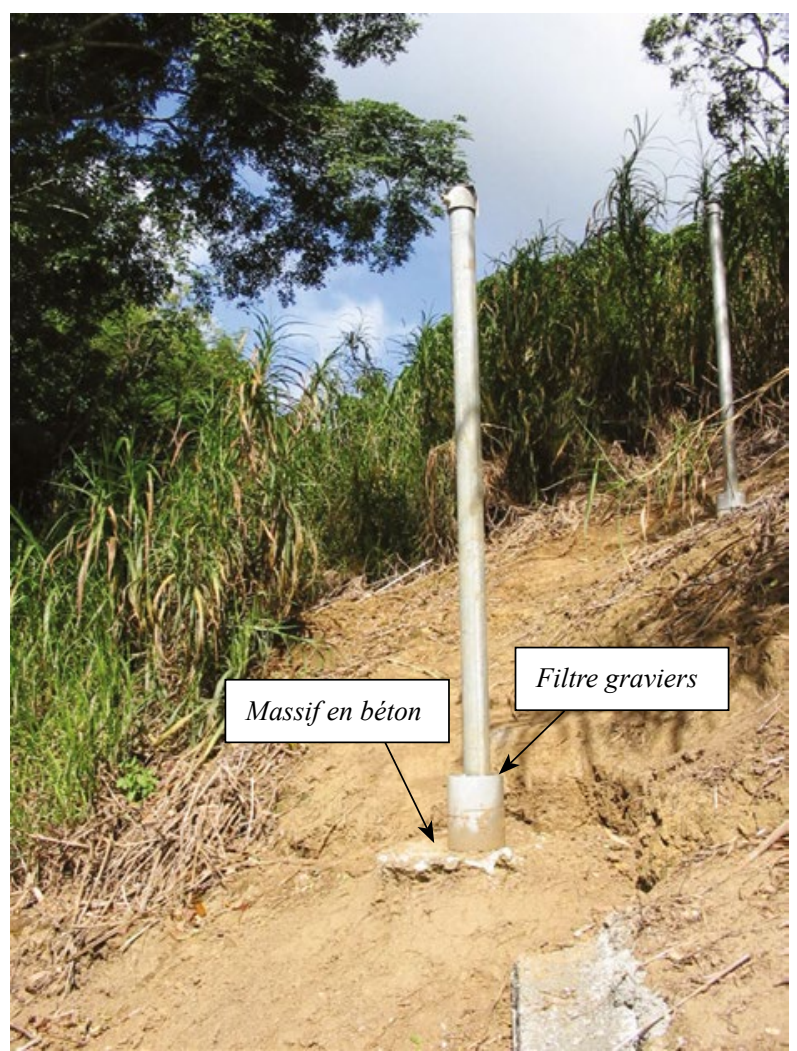

Figure 4 : Massif béton et filtre d'un CalédoMax.

Pour relever le niveau enregistré par un CalédoMax, il convient d'enlever le capot, puis de sortir la réglette sur laquelle sont tendues les cordes. Pour faciliter cette opération et éviter les risques de modifier la position de la bague, les CalédoMax possèdent en général une longueur ne dépassant pas 1,8 mètre. Ensuite, la distance entre la bague et l'extrémité supérieure de la réglette est mesurée. Enfin, la remise à zéro s'effectue simplement en remettant la bague dans la position basse, avant de remettre le système d'enregistrement dans le tube. Il ne faut donc que quelques minutes pour relever et remettre en service un CalédoMax. Grace à un nivellement préalable du sommet du CalédoMax, la cote maximale atteinte par la crue est ainsi facilement obtenue par une simple soustraction à la cote du sommet du CalédoMax de la distance précédemment mesurée, augmentée des 5 centimètres correspondants à la hauteur des flotteurs. Les solutions développées ainsi que les matériels constituant un CalédoMax rendent son coût très abordable. Bien que nous n'ayons plus réalisé de CalédoMax depuis plusieurs années, nous avons estimé son coût de revient actuel en Nouvelle-Calédonie à moins de $250 €$. En outre, les choix technologiques opérés le rendent réalisable à

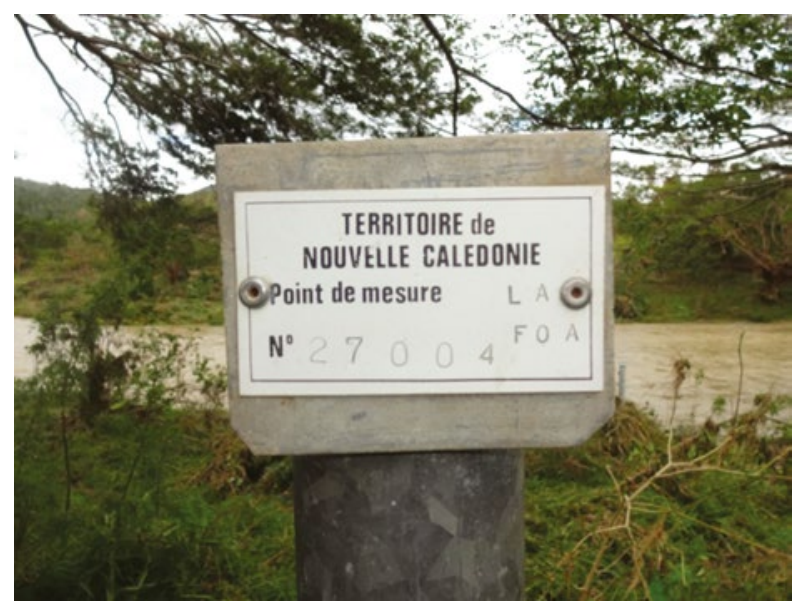

Figure 5 : Plaque signalétique d'un CalédoMax.

moindre frais dans des pays à faibles revenus, moyennant une adaptation avec les matériels effectivement disponibles.

\section{IMPLANTATION DES CALÉDOMAX}

Les CalédoMax sont classiquement installés en proximité des zones à enjeux (habitations, routes principales et ouvrages de franchissement, déversoirs...). Plusieurs appareils sont installés dans une section afin d'enregistrer les niveaux maxima atteints sur une très grande amplitude de hauteur d'inondation. L'information ainsi recueillie sert généralement à caler des modèles hydrauliques bâtis pour les études de définition des zones inondables.

Nous avons aussi installé des séries de CalédoMax au droit de nos stations hydrométriques. Cette redondance d'information sur la cote maximale atteinte en crue est très utile pour analyser les informations fournies par la station limnimétrique en cas de doute. Par exemple, lors de la crue majeure de novembre 2016 à Houaïlou, le massif de béton sur lequel était fixée la sonde ayant été déplacé de quelques mètres par la crue, l'enregistrement de la sonde toutefois disponible a pu être réanalysé à l'aide de l'information fournie par le CalédoMax, ce qui a permis à la DAVAR [2016] de proposer un hydrogramme reconstitué pour cette crue. Dans Nouvelot [1993] et OMM [2008], cet usage des échelles à maxima en complément des stations limnimétriques est clairement inventorié.

Une autre utilisation néo-calédonienne des CalédoMax pour l'hydrométrie consiste à installer plusieurs séries de CalédoMax à proximité d'une station limnimétrique afin de disposer de la pente de la ligne d'eau maximale d'une crue. En règle générale, une série de CalédoMax est disposée

Tableau 1 : Liste des événements significatifs et cotes de plus hautes eaux disponibles pour estimer les pentes des lignes d'eau en crue à la station de Kouaoua.

\begin{tabular}{|l|c|c|c|c|c|c|c|c|c|}
\hline $\begin{array}{c}\text { Profil en travers } \\
\text { de la vallée }\end{array}$ & $\begin{array}{c}\text { abscisses } \\
(\mathbf{m})\end{array}$ & $\begin{array}{c}\mathbf{2 8} / \mathbf{0 6} \\
\mathbf{2 0 0 4}\end{array}$ & $\begin{array}{c}\mathbf{0 8} / \mathbf{0 1} \\
\mathbf{2 0 0 5}\end{array}$ & $\begin{array}{c}\mathbf{2 2} / \mathbf{0 1} \\
\mathbf{2 0 0 6}\end{array}$ & $\begin{array}{c}\mathbf{2 5} / \mathbf{0 8} \\
\mathbf{2 0 0 7}\end{array}$ & $\begin{array}{c}\mathbf{2 0} / \mathbf{0 2} \\
\mathbf{2 0 0 8}\end{array}$ & $\begin{array}{c}\mathbf{2 2} / \mathbf{0 3} \\
\mathbf{2 0 0 8}\end{array}$ & $\begin{array}{c}\mathbf{2 4 / 0 4} \\
\mathbf{2 0 0 8}\end{array}$ & $\begin{array}{c}\mathbf{0 5} / \mathbf{0 2} \\
\mathbf{2 0 0 9}\end{array}$ \\
\hline CalédoMax (amont sonde) & 320 & 102,25 & 104,45 & - & 104,87 & 102,63 & - & 101,3 & 101,95 \\
\hline Hauteur maximale à la sonde & 220 & 102,08 & 104,18 & 100,38 & 104,5 & 102,49 & 103,25 & 101,14 & 101,77 \\
\hline CalédoMax (proche sonde) & 193 & 102,17 & 104,24 & 100,5 & 104,58 & 102,52 & 103,32 & 101,19 & 101,82 \\
\hline CalédoMax (aval sonde) & 100 & 101,89 & 103,97 & 100,24 & 104,34 & 102,3 & 103,07 & 100,92 & 101,6 \\
\hline
\end{tabular}




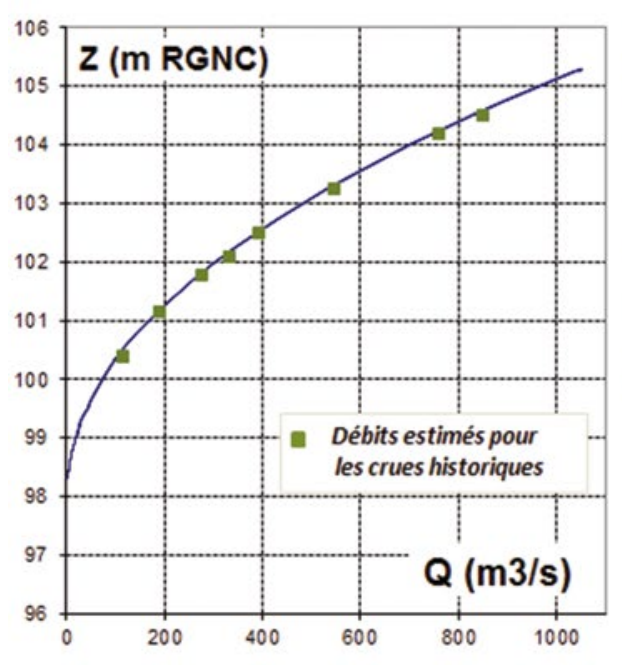

a) Courbe de tarage de la station.

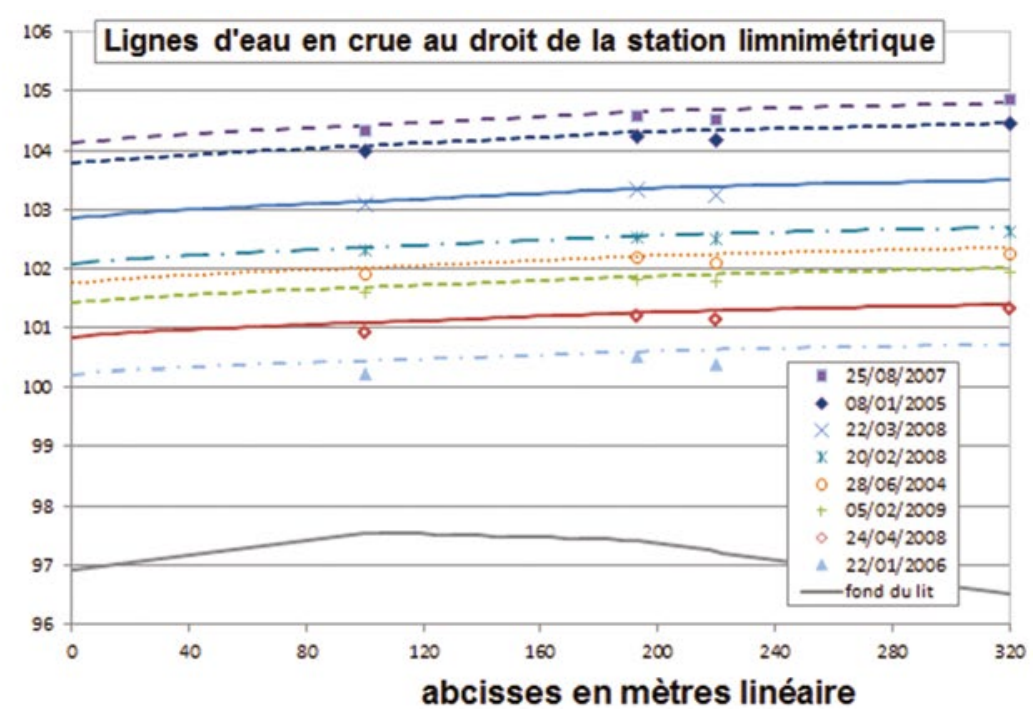

b) Profil en long des lignes d'eau modélisées en crue.

Figure 6 : Courbe de tarage et profils en long des lignes d'eau modélisées en crue à la station de Kouaoua.

dans une section de vallée, à quelques centaines de mètres en amont de la station et une autre à quelques centaines de mètres en aval. En supposant que le phénomène d'hystérésis est négligeable sur le bief où est installée notre station limnimétrique, ce qui est généralement le cas compte tenu des pentes topographiques existantes, il est alors possible d'estimer les pertes d'énergie par frottement sur cette portion de cours d'eau pour différents débits de crue. L'intégration de ces informations dans une modélisation hydraulique locale des crues permet alors d'extrapoler vers de plus forts débits la courbe de tarage.

Dans le cas de la station de Kouaoua présenté en figure 6, il n'existait pas de jaugeage en crue pour réaliser le calage du modèle hydraulique $1 \mathrm{D}$ que nous avons construit pour extrapoler la courbe de tarage présentée en figure $6 \mathrm{a}$. La nature des lits et l'analyse de sensibilité des résultats du modèle au choix du coefficient de Strickler, ont fourni une courbe de tarage médiane avec une incertitude d'environ $20 \%$ sur les valeurs de débit, les coefficients de Strickler retenus étant de $20 \mathrm{~m}^{1 / 3} / \mathrm{s}$ en lit mineur et de $15 \mathrm{~m}^{1 / 3} / \mathrm{s}$ en lit majeur. Ensuite, l'échantillon d'événements significatifs présenté dans le tableau 1 a été utilisé pour vérifier que les pertes de charges linéaires approchées via les séries de CalédoMax et celles estimées à l'aide du modèle étaient suffisamment similaires pour confirmer le choix des paramètres opéré. Ainsi, la courbe de tarage retenue a été utilisée pour estimer les débits des crues historiques, puis ceux-ci ont ensuite été utilisés dans la modélisation hydraulique afin de reconstituer les lignes d'eau au droit de la station pour chacune de ces crues. La bonne cohérence entre les lignes d'eau modélisées et celles issues des CalédoMax (figure $6 \mathrm{~b}$ ) a permis de considérer cette courbe de tarage comme la meilleure possible, compte tenu des informations disponibles. Finalement, issue d'un nombre très limité de profils topographiques relevés in situ (4 profils) et des informations disponibles aux 3 séries de CalédoMax, cette proposition de courbe de tarage nous semble disposer d'une robustesse déjà intéressante jusqu'à des débits de crue de période de retour de l'ordre de 5 ans. Bien entendu, l'amélioration de cette courbe de tarage passera d'une part par l'exploitation des informations issues des CalédoMax pour les prochaines crues plus rares et si possible par des jaugeages effectués lors de grandes crues.
Lors de notre recherche bibliographique, une dernière utilisation des échelles à maxima a été recensée. Elle consiste à implanter une échelle à maxima au droit d'ouvrages (seuil, déversoir, petits ouvrages de franchissement) pour lesquels la relation entre le débit et la hauteur d'eau est facile à approcher. Cette technique est généralement utilisée afin d'estimer les débits de crue sur de très petits bassins versants, comme dans Piégay et al. [2006] où l'échelle à maxima est associée un déversoir auto-jaugeur. Plusieurs séries d'échelles à maxima permettent aussi l'estimation des débits de crue via les pentes des lignes d'eau maximales?

\section{RETOUR D'EXPÉRIENCE DE L'UTILISATION DES CALÉDOMAX}

Les premiers CalédoMax ont été installés en 1987/1988, et les premières mesures ont été effectuées suite à l'épisode cyclonique Anne qui a touché la Nouvelle-Calédonie aux environs du 13 janvier 1988. Depuis 1988, 918 CalédoMax ont ainsi été installés, et à ce jour encore 692 sont encore actifs, répartis sur l'ensemble de la Grande Terre (figure 7). En tout, ce sont plus de 16400 mesures qui ont été effectuées en près de 30 ans.

Les CalédoMax montrent une excellente résistance aux intempéries et à la corrosion, malgré des installations dans des environnements hostiles (air salin des régions côtières, forte humidité, eaux chargées en minéraux) qui favorisent l'oxydation. L'utilisation d'aciers exclusivement galvanisés ou encore de composants en matière plastique destinés au milieu marin participent de cette robustesse.

Les comparaisons entre les niveaux maxima de crues estimés à l'aide des CalédoMax et ceux issus des sondes montrent souvent une très bonne corrélation. Les écarts entre ces niveaux peuvent provenir de la méthode de mesure retenue dans les CalédoMax qui approche la charge hydraulique, mais aussi du lieu de la mesure. En effet, les niveaux de crue en lit majeur là où les CalédoMax sont installés, peuvent être différents du niveau de crue dans le lit mineur

7. https://mo.water.usgs.gov/surfwat/CSGWeb/, consulté le 9/05/2017 


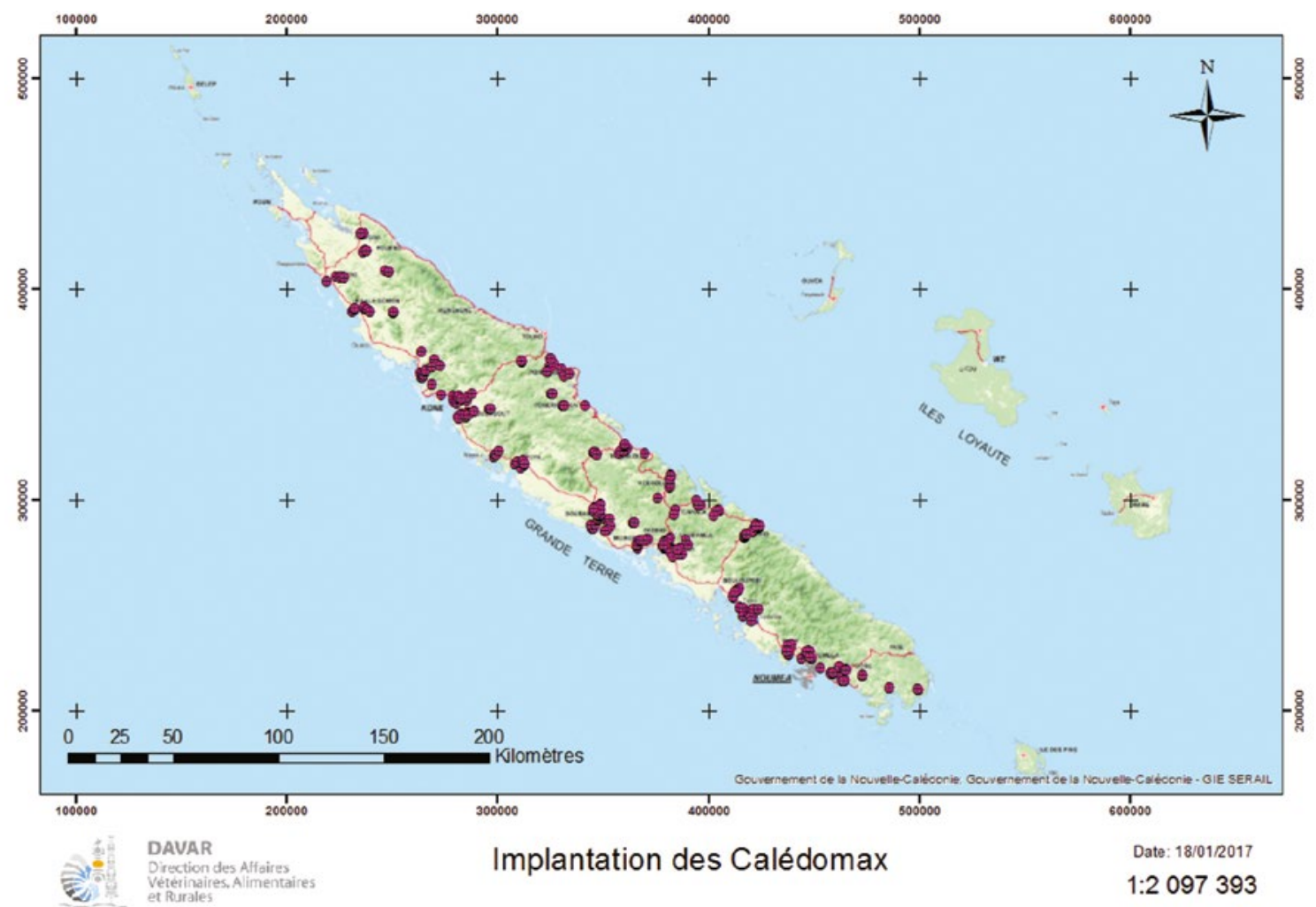

Figure 7 : Implantation des CalédoMax en Nouvelle-Calédonie.

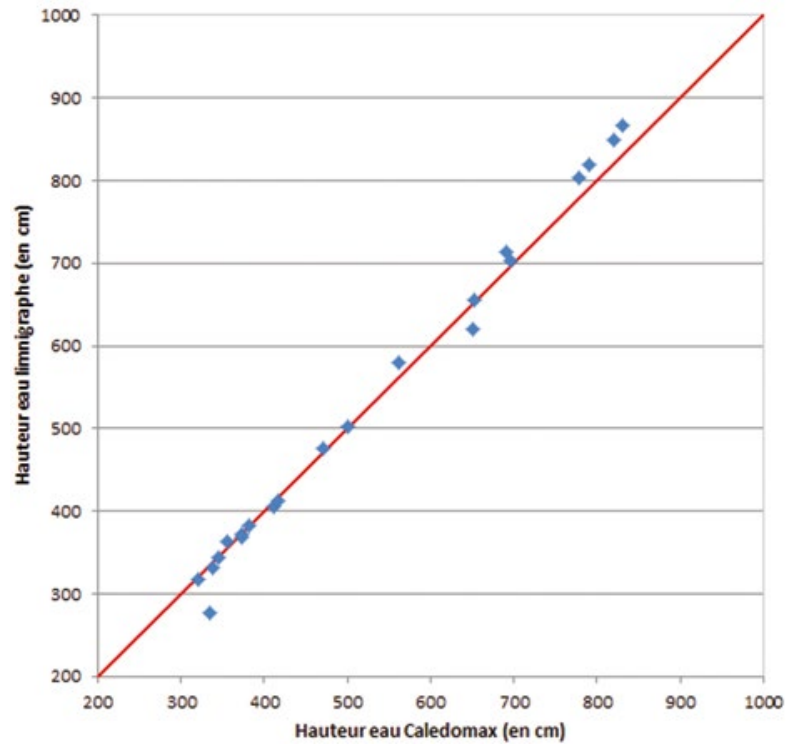

Figure 8 : Comparaison des hauteurs maximales issues des CalédoMax et celles issues de la sonde limnimétrique à la station de Boutana.

où les sondes sont installées. L'analyse complète et exhaustive de ces comparaisons reste toutefois à mener.

Les dysfonctionnements sont rares, et sont le plus souvent dus à des causes exogènes telles que le vandalisme ou encore les feux de brousse qui font fondre le système de mesure des CalédoMax. Dans de rares cas la présence dans le tube de nids de fourmis ou de geckos (lézards) au pied des réglettes, ou le colmatage du filtre gravier par la boue ont pu perturber le déplacement du flotteur. Nous avons aussi recensé la possibilité de casse des fils dans la réglette ou encore la rouille de la réglette dans le tube empêchant une mesure correcte.

Au $1^{\text {er }}$ juillet 2017, 544 «Mesures impossibles » ont été recensées, qui s'expliquent par diverses raisons : dysfonctionnement de l'appareil, vandalisme, impossibilité d'accès... Leur nombre a fortement augmenté depuis 2012 : 251 entre 2012 et 2017 ( 5 ans), contre 293 mesures impossibles recensées entre 1990 et 2012 (22 ans). L'entretien et la relève des CalédoMax ont été annuellement effectués par un agent connaissant parfaitement le réseau et ce jusqu'au début de l'année 2012. Depuis, ils sont réalisés en fonction des disponibilités des équipes de terrain en charge des mesures hydrométriques, ainsi que de la survenue de crues significatives. En conséquence, le nombre d'échec de géolocalisation de CalédoMax, même avec un GPS, et le nombre de fois où les équipes ont trouvé des accès fermés aux propriétés privées ont largement augmenté (115 fois depuis 2012).

Les tournées régulières permettent d'éviter que les appareils soient détruits ou démontés, assurent que les réglettes soient en bon état de fonctionnement le jour où cela est nécessaire et contribuent à ce que les accès restent disponibles pour les agents de terrain. La régularité dans les visites rassure les propriétaires, qui sont au fait de ce passage habituel et ne sont pas étonnés de voir des étrangers sur leur propriété quelques jours à peine après les épisodes de crues.

Pour assurer leur longévité, il convient de changer périodiquement les filtres de graviers des CalédoMax car ceux-ci peuvent se colmater. Et comme la plupart des appareils de mesure installés sur le terrain, il convient de réaliser au moins une visite annuelle, de préférence avant la saison la plus sujette aux crues, afin de vérifier que le système de mesure de la hauteur d'eau reste opérationnel et, si besoin, 
le remettre à zéro. Lors de ces visites, on s'assure en général que le déplacement du flotteur et le maintien de la bague dans sa position maximale sont fonctionnels.

Enfin, comme pour la plupart des systèmes d'échelles, les risques de détérioration par les crues voire de perte, existent pour les CalédoMax. Ces risques sont dus principalement à des heurts provenant des corps flottants (troncs, branches,...) ou encore par leur accumulation sur l'appareil augmentant ainsi la surface en prise avec l'écoulement. Le cas échéant, le système de fixation du tube cylindrique dans l'embase par boulon et une cheville permet un remplacement rapide. 214 CalédoMax ont disparu suite aux crues ou ont été désinstallés ou non remplacés suite à une détérioration.

\section{ORGANISATION DES INFORMATIONS FOURNIES PAR LES CALÉDOMAX}

L'ensemble des mesures des CalédoMax installés en Nouvelle-Calédonie ont été saisies et stockées dans une base de données Access, dénommée BdD_PHE (base de données Plus Hautes Eaux). Cette base de données regroupe non seulement les mesures issues des CalédoMax mais aussi les relevés de laisses de crue réalisés lors d'enquêtes dédiées ou lors des études de définition des zones inondables.

La principale utilisation de cette base de données est un rôle d'archivage et de suivi de la maintenance des CalédoMax. La base de données conserve les numéros et les emplacements (coordonnées GPS) des appareils, ainsi que leur état lors de la dernière visite terrain, les travaux et opération d'entretien ou de maintenance à prévoir et les coordonnées de propriétaires de terrain lorsque nécessaire. La seconde utilisation de cette base de données est l'export des mesures pour les études nécessitant le calage de modèles hydrodynamiques. L'export de la BdD_PHE se fait au format tableur Excel, avec à ce jour 4 formats possibles : métadonnées, mesures, tournées à prévoir et contacts. Des mises en forme spécifiques sont créées dans Excel pour faciliter la transformation en fichier SHAPE et SIG.

Les métadonnées de cette base de données sont disponibles sur internet, sur le site dédié aux informations sur l'eau de Nouvelle-Calédonie, l'Explo Cart'Eau (www.davar.gouv.nc/ explocarteau). Il s'agit principalement du type de source de la mesure (CalédoMax, laisses de crues ponctuelles ou enquêtes dédiées), de la date d'installation du CalédoMax et du nombre de mesures disponibles au point considéré. Ces métadonnées permettent notamment aux bureaux d'études ou aux particuliers de connaître les mesures disponibles dans un secteur particulier et de se rapprocher de la DAVAR pour obtenir les données les plus à jour sur ce secteur.

\section{CONCLUSION}

L'estimation, en des endroits préalablement définis, des hauteurs maximales atteintes par les crues est une nécessité pour l'activité d'observation des rivières de Nouvelle-Calédonie réalisée par la DAVAR. Et ce, tant pour l'acquisition des données hydrométriques que pour le développement de modèles hydrauliques des crues. Cette nécessité nous a conduit à développer un instrument de mesure adapté à notre contexte géo-climatique, les CalédoMax, à en implanter près de 1000 sur l'ensemble de la Grande Terre et à développer une base de données permettant leur exploitation rapide et leur valorisation.

Les CalédoMax sont, comme la majorité des échelles à maxima que nous avons recensées, munis d'une enceinte cylindrique permettant de protéger le système de mesure des éventuelles perturbations extérieures. Les doutes sur d'éventuelles interventions humaines post-crues, sont ainsi inexistants, ce qui laisse une plus grande liberté dans le délai de relève des appareils, la contrainte la plus importante devenant alors le risque de survenue d'une seconde crue et par là l'impossibilité de savoir à quelle crue se rattache la hauteur relevée. Fréquemment, nous opérons des relevés de CalédoMax plusieurs semaines voire plusieurs mois après le passage d'une crue, sans que l'information recueillie ne soit dégradée.

Pour disposer du plus grand nombre de sites possibles équipés de CalédoMax, les coûts de fabrication, d'entretien et de relève de ces appareils ont été voulus les plus réduits possibles. En outre, la simplicité de conception les rend accessibles à tout opérateur disposant d'un atelier de mécanique rudimentaire permettant le découpage, le pliage et la soudure d'aciers galvanisés. De la même manière l'installation in situ est aisée et l'éventuel remplacement rapide à effectuer. La robustesse et la solidité de ces appareils ont aussi été démontrées par le faible taux de remplacement que nous avons observé comme par le peu de mesures erronées remarquées. Avec un protocole de relève plutôt simple et rapide, ces appareils nous semblent particulièrement adaptés pour les pays à faibles revenus.

\section{REMERCIEMENTS}

Les auteurs de l'article souhaitent remercier les personnels de la DAVAR qui ont contribué de près ou de loin à la mise au point des CalédoMax. Nous tenons en particulier à rendre un hommage marqué à Christian DELEBRET, Gilles RICAUD et Bernard REY qui sont les concepteurs de ce système ingénieux de mesure des hauteurs maximales atteintes lors des crues.

\section{RÉFÉRENCES}

Davar (2016) - Bilan De La Crue Du 22 Novembre 2016. ftp://ftp.gouv.nc/DAVAR/Etudes_GP/Hydrologie/Bilans_Hydro/

FRIDAY J. (1965) — Tests of crest-stage intake systems. USGS open-file report USGS OFR65-59A.

Nouvelot J.F. (1993) — Guide des pratiques hydrologiques sur les petits bassins versants ruraux en Afrique tropicale et équatoriale. Édition IRD - CIEH, 44-45.

Omm, Organisation Mondiale De La Meteorologie (2008) Guide des pratiques hydrologiques, volume numéro 168 : I-5-1, 168 : I-5-1.

Piegay H., Landon N., Liebault N. (2006) — LiFE Eau et Forêt - Rapport de la composante française : Synthèse des opérations de suivi des sites-pilotes de l'Ouvèze et de la Drôme, version finale du 31 décembre $2006: 12$.

Piotte O., Pasquet F., Chaleon C., Chambon D., Thomas L., Souldadie J.L., Perherin C., Galiana C. (2016) — Vers une gestion collaborative des recensements de repères de crues, La Houille Blanche, 4 : 11-17.

Roose E. (2000) - Gully erosion in Africa. In Gully erosion under global change, Leuven, Belgium, 16- 19 April, abstract 111.

UsGS (2010) - Stage measurement at gaging stations, chapter 7 of book 3, section a, techniques and methods 3-a7, Book 3, 17-18.

Zaher H, Cours de Conservation Des Sols Et De L'eau Agence Universitaire De La Francophonie, http://www. ma.auf.org/erosion/index.html (2017) — Cours de conservation des sols et de l'eau - agence universitaire de la francophonie, http://www.ma.auf.org/erosion/index.html. 